\title{
Missense Mutation
}

National Cancer Institute ( $\mathrm{NCl})$

\section{Source}

National Cancer Institute (NCI). Missense Mutation.

A genetic alteration in which a single base pair substitution alters the genetic code in a way that produces an amino acid that is different from the usual amino acid at that position. Some missense variants (or mutations) will alter the function of the protein. Also called missense variant. 